\title{
MAGNETIC DESIGN OF A SUPERCONDUCTING AGS SNAKE*
}

\author{
R. Gupta ${ }^{\#}$, A. Luccio, G. Morgan ${ }^{+}$, W. Mackay, K. Power, T. Roser, E. Willen, \\ Brookhaven National Laboratory, Upton, NY 11973 USA \\ M. Okamura, RIKEN, Saitama, Japan
}

\section{Abstract}

Brookhaven National Laboratory plans to build a partial helical snake for polarized proton acceleration in the AGS. It will be a 3 Tesla superconducting magnet having a magnetic length of 1.9 meter. AGS needs only one magnet and currently there is no plan to build a prototype. Therefore, the first magnet itself must function at the design operating field and provide the required field quality, spin rotation and deflections on the particle beam. New software have been developed that exchanges input/output between the OPERA3d field design program, the Pro-Engineering CAD model and the software that drives the machine to make slots in aluminum cylinders where blocks of 6-around-1 NbTi wires are placed. This new software have been used to carry out a number of iterations to satisfy various design requirements and to assure that the profile that is used in making field computations is the same that is used in cutting metal. The optimized coil cross-section is based on a two layer design with both inner and outer layers having five current blocks per quadrant. The ends are based on a design concept that will be used for the first time in accelerator magnets.

\section{INTRODUCTION}

At present there is a significant loss of polarization in accelerating polarized protons from $5 \mathrm{GeV}$ to $25 \mathrm{GeV}$ in Brookhaven AGS (Alternating Gradient Synchrotron). The proposed 30\% partial Siberian Snake can overcome both imperfection and intrinsic resonaces [1]. Drawing on our previous experience with superconducting helical magnets for RHIC [2], a $203.2 \mathrm{~mm}$ (8 inch) coil aperture helical dipole is being designed and built for AGS. The basic design parameters are listed in Table 1. The dipole field rotates with a pitch of 0.2053 degrees $/ \mathrm{mm}$ for 786 $\mathrm{mm}$ in the center and a pitch of 0.3920 degrees $/ \mathrm{mm}$ for $577 \mathrm{~mm}$ in each end. In order to minimize the residual deflections and offsets of the beam on its orbit through the Snake, a careful balancing of the coil parameters is necessary. This required the development of new software that apart from doing various calculations can also generate appropriate input/output for other $2 \mathrm{~d}$ and $3 \mathrm{~d}$ field calculations programs and for various CAD programs. Similarly a significant effort was also invested in developing and automating CAD based design techniques [3]. A good design for the coils was found after several iterations where the coil pitch and length of helical

\footnotetext{
* Work supported by the U.S. Department of Energy under Contract No. DE-AC02-98CH10886 and by RIKEN of Japan.

\#gupta@bnl.gov.

'Retired from BNL.
}

sections were optimized while retaining the cross section and ends. In addition to the main helical coils, a solenoid winding is planned inside the main coils to compensate for the axial component of the field that is experienced by the beam when it is off-axis in this helical magnet. Also, the magnet system will have several corrector magnets placed on the same tube on which the solenoid is placed. The engineering design of this magnet is presented elsewhere [3].

Table 1: AGS Helical Magnet Design Parameters.

\section{Superconductor Parameters:}

$\begin{array}{ll}\text { Filament diameter } & 10 \text { micron } \\ \text { Wire diameter } & 0.33 \mathrm{~mm} \\ \text { Cu to Non-Cu ratio } & 2.5: 1 \\ \text { Cable type } & 6 \text {-around-1 } \\ \text { Cable diameter, bare } & 0.99 \mathrm{~mm} \\ \text { Cable diameter, insulated } & 1.09 \mathrm{~mm} \\ \text { Cable } \mathrm{I}_{\mathrm{c}} @ 5 \mathrm{~T}, 4.2 \mathrm{~K} & 530 \mathrm{~A}\end{array}$

\section{Coil Parameters:}

No. of coil layers

Coil inner radius for inner layer

Coil inner radius for outer layer

Current blocks per quadrant

No. of turns in 9 blocks

No. of turns in inner-pole block

Other Parameters:

Design field

Quench field

Operating current

Quench current

Operating temperature

Stored energy@3T

Inductance

Pitch in the middle $(786 \mathrm{~mm})$

Pitch in the ends (577 mm each)

Slot size, width/depth

Warm bore tube id/od

Cold bore shield id/od

Cold bore tube id/od

Inner Aluminum tube id/od

Outer Aluminum tube id/od

Iron yoke id/od

Shell id/od

End plate thickness

Magnetic length

Aluminum tube length

Vacuum vessel length

2

$101.6 \mathrm{~mm}$

$127.8 \mathrm{~mm}$

10 ( 5 per layer)

$12 \times 9=108$

$12 \times 5=60$

$3.0 \mathrm{~T}$

$\sim 4.1 \mathrm{~T}$

$\sim 350 \mathrm{~A}$

$\sim 500 \mathrm{~A}$

$4.5 \mathrm{~K}$

$0.4 \mathrm{MJ}$

$6.5 \mathrm{H}$

$0.2053 \mathrm{deg} / \mathrm{mm}$

$0.3920 \mathrm{deg} / \mathrm{mm}$

$13.6 / 13.1 \mathrm{~mm}$

$152.4 / 156.5 \mathrm{~mm}$

$165.2 / 167.7 \mathrm{~mm}$

$176.5 / 181.6 \mathrm{~mm}$

$195.6 / 229.6 \mathrm{~mm}$

$248 / 281.8 \mathrm{~mm}$

$300.4 / 685.8 \mathrm{~mm}$

$685.8 / 687.1 \mathrm{~mm}$

$12.7 \mathrm{~mm}$

$1.9 \mathrm{~m}$

$2.27 \mathrm{~m}$

$2.57 \mathrm{~m}$

\section{2-D MAGNETIC DESIGN}

The cross section of a helical coil may be specified in a plane that is perpendicular to the helix and with a pitch by 
which the field is required to rotate. In the present design, the pitch is different in the middle of the dipole as compared to that near the two ends (see Table 1). Thus the cross section of a coil cut in a plane perpendicular to the axis of the magnet will be different as compared to the helical cross section and also it will be different in the two regions with different pitches. The design procedure developed here is such that the initial coil cross section is optimized for a good field quality in a straight dipole magnet. The size of conductor blocks in this straight dipole is the same as in the plane perpendicular to the helix. This means that in a plane perpendicular to the beam axis the subtended block size is different for two pitches and for two layers. The magnetic design of this straight magnet, where for the same helical block cross section, the separation between the blocks is much larger than that it is in the helical magnet, is shown in Fig. 1.

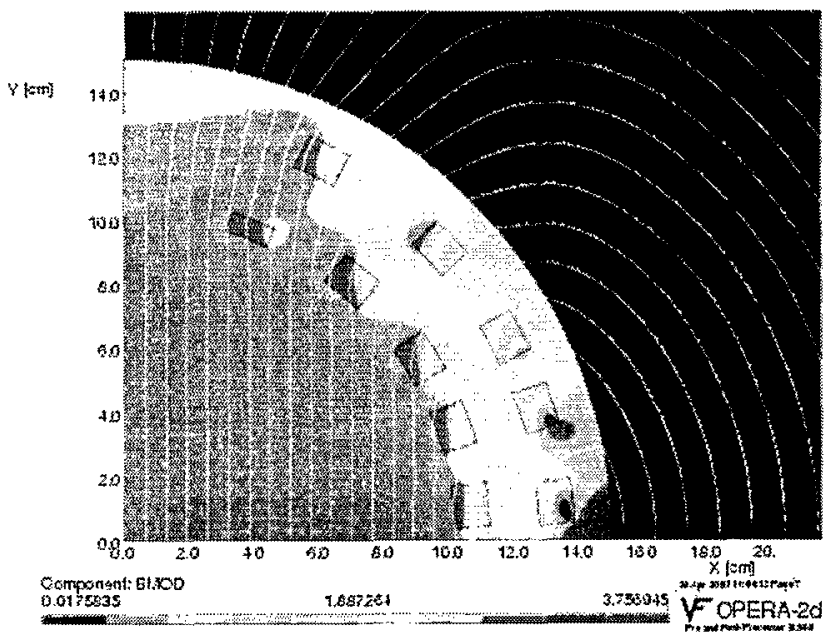

Fig. 1: Cross section of the straight magnet with the identical block size as in helical section.

The center of each block retains the same relative position as in the helical section. The optimized coil cross section consists of five conductor blocks per quadrant in each of two layers. To minimize the design effort, the coil is optimized such that all slots in the two aluminum cylinders have an identical cross section in the plane perpendicular to the helix. In addition, all blocks, except the pole block in the inner layer, consist of nine layers each of 12 turns (a total of 108 turns per block). To reduce the peak field on the conductor, the pole slot in the aluminum tube for the inner layer is filled with five instead of nominal nine layers. The above adjustment in the number of layers in a block can also be used to partially iterate the field quality without changing the detailed design even after the slots have been machined in the aluminum tube. This method was first used successfully in RHIC helical dipole magnet [2].

In a straight magnet with no axial component of the field, the field quality is expressed in terms of the normal and skew harmonics, $b_{n}$ and $a_{n}$, (magnitude $c_{n}$ with $c_{n}{ }^{2}=a_{n}^{2}+b_{n}^{2}$ ) defined in the following expansion of horizontal and vertical components of field $\left(B_{x}, B_{y}\right)$ :

$$
B_{y}+i B_{x}=10^{-4} \times B_{o} \sum_{n=0}^{\infty}\left(b_{n}+i a_{n}\right)[(x+i y) / R]^{n}
$$

where $x$ and $y$ are the horizontal and vertical coordinates, $B_{0}$ is the dipole field strength and $R$ is the "reference radius" that is chosen to be $65 \mathrm{~mm}$ here. The computed harmonics at $1 \mathrm{~T}$ and $3.1 \mathrm{~T}$ are given in Table 2.

Table 2: Computed values of normal harmonics $\left(b_{n}\right)$ at $1 \mathrm{~T}$ in a 2-d model where coils are straight.

\begin{tabular}{|l|c|c|c|c|c|}
\hline Harmonic No. & 2 & 4 & 6 & 8 & 10 \\
\hline Harmonics@1 T & 0.0 & -0.1 & -0.4 & -3.1 & 1.3 \\
\hline Harmonics@3T & 2.2 & -1.4 & -0.5 & -3.1 & 1.3 \\
\hline
\end{tabular}

\section{3-D MAGNETIC DESIGN}

Fig. 2 shows the OPERA3d model of the coils with the magnitude of field superimposed on the surface of conductor blocks for a field of $3.12 \mathrm{~T}$ at the center of the magnet. These calculations are done with the iron yoke. The peak field, which is used in computing the quench field in Table 1, is $3.86 \mathrm{~T}$.

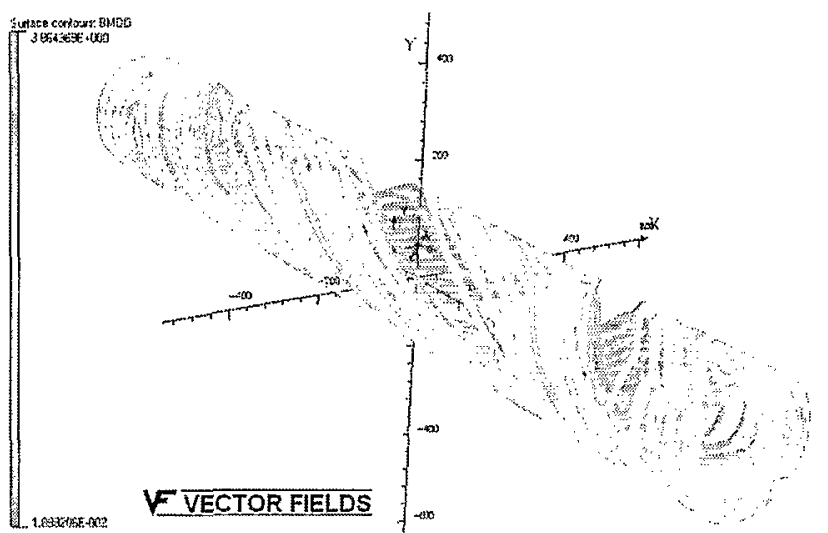

Fig. 2: OPERA3d model of the coils with the magnitude of the field superimposed on the conductor surface. The field at center is $3.12 \mathrm{~T}$ and the iron yoke is not shown for clarity.

The conventional 2-d field harmonics description for a straight magnet is not valid in the case of helical fields. The harmonics become dependent on integration radius and on helical pitch. The harmonic computation, however, can still be used in describing fields in limited sense, when properly used. In Fig. 3, the sextupole harmonics are plotted as a function of axial position by integrating $B_{y}$ at a radius of $65 \mathrm{~mm}$. The field in the center of the magnet is $3.12 \mathrm{~T}$.

The end design is based on the formalism developed by G. Morgan [4]. As compared to RHIC snake [2], we have improved the end design as (a) the helical rotation of the coil continues through the end and (b) the bend radius is significantly reduced to increase the effective field generated for a specified coil length. The prescription by Morgan minimizes harmonic content by a judicious choice of angles of the current blocks as they traverse side-to-side in the ends. An end design similar to that used in the RHIC helical magnets was also developed [5]. 
However, the current Morgan end design was preferred for its harmonic content and efficiency.

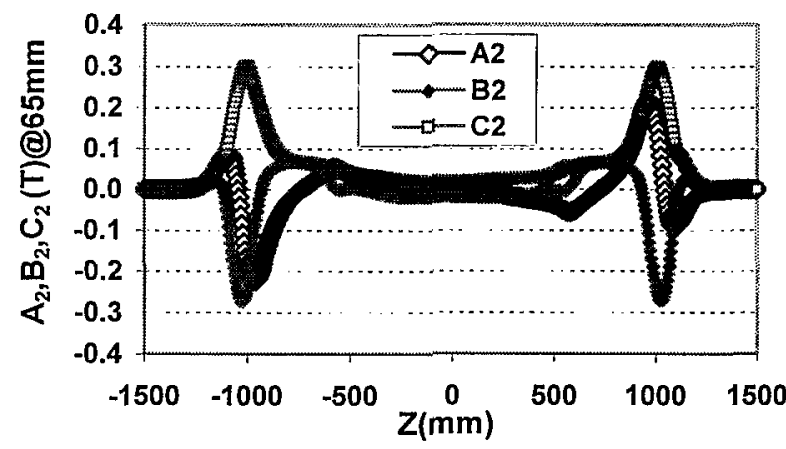

Fig. 3: The sextupole harmonic along the magnet axis computed by integrating $B_{y}$ at a radius of $65 \mathrm{~mm}$ and using the harmonic definitions appropriate for 2-d fields.

The magnitude of the field and the field components on the magnet axis are shown in Fig. 4. The change in the magnitude of the field in the magnet center and near two ends is caused by the difference in the pitch by which the magnetic field is required to rotate.

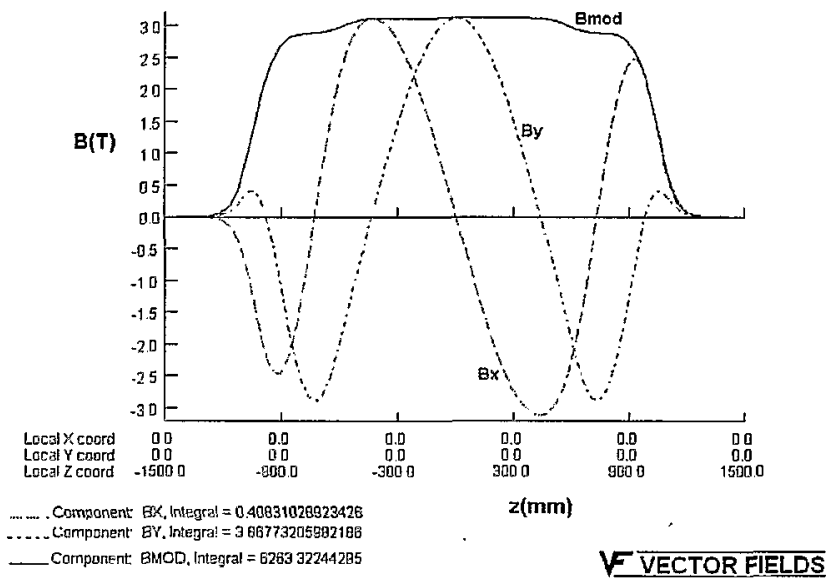

Fig. 4: The magnitude and the components of field on the magnet axis.

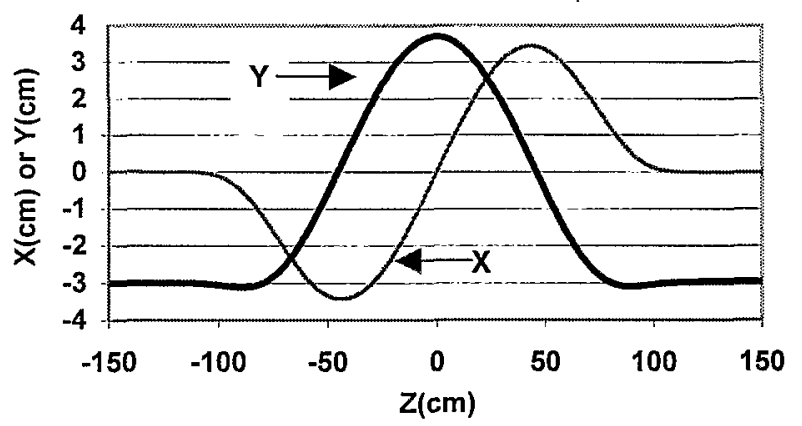

Fig. 5: The nominal horizontal and vertical position of the beam as function of axial position inside the helical magnet.

The actual beam is injected $3 \mathrm{~cm}$ off-axis in the horizontal plane to maximize the use of available aperture. The field inside the helical magnet must satisfy some stringent requirements. Apart from satisfying the spin dynamics requirements, the beam must exit the helical magnet with nearly the same position and angle by which it entered the magnet. This requirement was satisfied after a number of involved iterations. This iteration process benefited by the development of various in-house software packages and techniques. The final results are shown in Fig. 5 where the horizontal and vertical position of the beam is plotted as the function of axial position in the helical magnet.

The beam experiences an axial component of the field when it is away from the magnet axis. In the present design, the net integral of this axial component, as experienced by the beam along the path shown in Fig. 5, has a significant non-zero value. One way to compensate this is by introducing an additional solenoidal coil in the middle of the magnet. This is shown in Fig. 6. The other corrector windings will be placed on the same tube near the ends.

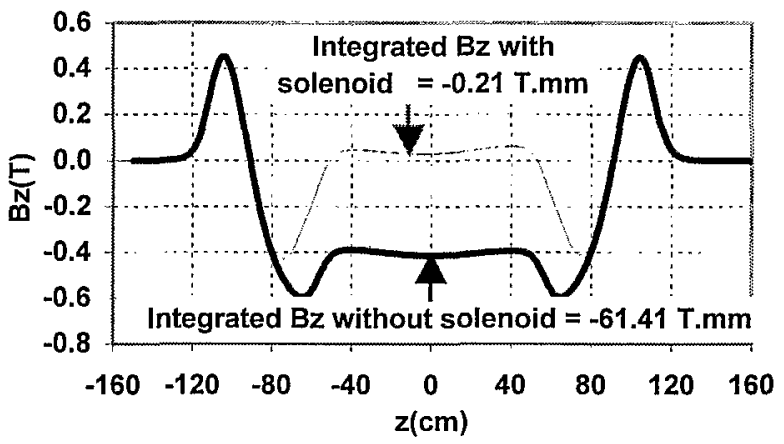

Fig. 6: The axial component of the field along the nominal beam path. The integral value is made zero with the help of a solenoidal winding on the beam tube in the middle of the magnet.

\section{SUMMARY}

The design and analysis of a partial helical snake for AGS has been completed. A number of software techniques have been developed to obtain a design that satisfies the basic requirements.

\section{ACKNOWLEDGMENTS}

We would like to thank M. Anerella, M. Harrison, P. Kovach, A. Marone and S. Plate for their constant support and encouragement.

\section{REFERENCES}

[1] T. Roser, et al., "Acceleration of Polarized Beams using a Strong Partial Siberian Snake", this conference.

[2] E. Willen, et al., "Performance Summary of the Helical Magnets for RHIC", this conference.

[3] M. Anerella, et al., "Engineering of AGS Snake coil Assembly", this conference.

[4] G. Morgan, "Private Communication".

[5] M. Okamura, et al., "Design Study of A Partial Snake for AGS", Proceedings of EPAC 2002. 